\title{
Annually Radiographic Periodontal Bone Loss Rates of Tooth Affected Severe Advanced Periodontitis with Secondary Occlusal Traumatism
}

\author{
Guey-Lin Hou ${ }^{1,2 *}$ \\ ${ }^{1}$ Former Professor, Graduate Institute of Dental Sciences and Department of Periodontics, School of Dental Medicine, Kaohsiung Medical \\ University, Kaohsiung City, Republic of China \\ ${ }^{2}$ Former Chairman and Professor, Dental Department, Periodontal Prosthetic Center, Chang-Gung Memorial Hospital, Kaohsiung City, Taiwan, \\ Republic of China
}

*Corresponding author: Guey-Lin Hou, Former Professor, Graduate Institute of Dental Sciences and Department of Periodontics, School of Dental Medicine, Kaohsiung Medical University, Kaohsiung City, Republic of China, Tel: 0088677472606; E-mail: hougl2001@yahoo.com.tw

Received: 14 Sep, 2020 | Accepted: 23 Sep, 2020 | Published: 30 Sep, 2020

Citation: Hou GL (2020) Annually Radiographic Periodontal Bone Loss Rates of Tooth Affected Severe Advanced Periodontitis with Secondary Occlusal Traumatism. Int J Dent Oral Health 6(6): dx.doi.org/10.16966/2378-7090.339

Copyright: (c) 2020 Hou GL. This is an open-access article distributed under the terms of the Creative Commons Attribution License, which permits unrestricted use, distribution, and reproduction in any medium, provided the original author and source are credited.

\begin{abstract}
Objective: The purpose of present study was to evaluate the differences of teeth using annually radiographic alveolar bone loss in cases involved severe advanced periodontitis with secondary occlusal traumatism among at the baseline of periodontal evaluation.

Materials and methods: A total 1013 tooth of 53 individuals affected severe advanced periodontitis with secondary occlusal traumatism was examined annually radiographical periodontal bone loss for maxillary and mandibular molars and non-molars teeth with and without secondary occlusal traumatism. The annually radiographical periodontal bone loss of teeth affected severe advanced periodontitis with secondary occlusal traumatism were calculated using the digital scanning radiographic image analysis.

Results: Results showed 1) that he reliability coefficient of interexaminer and intraexaminer measurements of ARABL of teeth for maxillary, mandibular and both arches of teeth with SAP using DSRIA indicated a statistically significant difference $(p<0.001)$ using two-sample t-test; 2$)$ that differences of ARABL ( $\mathrm{mm} / \mathrm{yr}$.) in maxillary and mandibular teeth with SOT and without SOT in Individuals, respectively, and showed a statistically significance $(\mathrm{p}<0.0001)$ using two-sample t-test; 3$)$ that the differences of ARABL $(\mathrm{mm} / \mathrm{yr}$.) on molars and non-molars with and without SOT in maxillary and mandibular arches, respectively, and showed a statistically significant difference $(p<0.001)$ using two-sample t-test; 4$)$ that the differences of ARABL $(\mathrm{mm} / \mathrm{yr}$.) on non-molars with and without SOT in both the maxillary and mandibular arches, respectively, and presented a significant difference
\end{abstract} $(p<0.0001)$ using two-sample t-test.

Conclusion: We concluded that the means of annually radiographic alveolar bone loss on teeth affected severe advanced periodontitis with secondary occlusal traumatism were significantly greater losses than the teeth without secondary occlusal traumatism, irrespective of maxillary and mandibular molars or non-molars.

Clinical relevance: This is the first report to address the periodontal bone lose rate per year of naturally progressing severe advanced periodontitis affected secondary occlusal traumatism using the digital scanning radiographic image analysis in Taiwanese subjects at the baseline data.

Keywords: Annually radiographical periodontal bone loss; Severe advanced periodontitis; Secondary occlusal traumatism

\section{Introduction}

The majority of the cross-sectional and longitudinal studies employing techniques relating to measuring the rate of periodontal attachment loss in individuals affected healthy periodontal, and different disease types, such as chronic periodontitis (CP), early-onset periodontitis (EOP), and aggressive periodontitis [1].

It is well known that there is little or no documents regarding studies were presented that the reduction in alveolar bone crest height with increasing age in healthy and in those with periodontal disease [2-6]. However, wide variations were found at the different degrees of proximal bone loss within the same mouth of the patients and among different types of periodontitis $[1,2,7,8]$. Normally, most studies employed periodontal probing and radiographic measurements to investigate the rate of periodontal attachment levels and radiographic bone loss.

Secondary occlusal trauma (SOT) (Figure 1) is injury resulting in tissue changes from normal or excessive occlusal forces applied to a tooth or teeth with reduced periodontal support. It occurs in the presence of attachment loss, bone loss, and normal/recessive occlusal force(s). The most common clinical sign of SOT to the periodontium 
is increased tooth mobility. In the injury stage of SOT there is destruction of periodontal fibers, which will increase the mobility of the tooth. In the final stage the accommodation of the periodontium to increased forces entails a widening of the periodontal ligament, which also leads to increased tooth mobility. When it becomes progressively worse, it can be considered pathologic. Other causes of increased tooth mobility include advanced bone loss, inflammation of the PDL of periodontal or periapical origin [9].

Little or limited information concerning yearly bone loss rates in the long-term studies of periodontal treatment outcomes in individuals with severe advanced periodontitis (SAP) with secondary occlusal traumatism (SOT) on the tooth at the baseline.

The purpose of this study was to investigate the rates of annually radiographic alveolar bone loss (ARABL) on the teeth affected SAP with SOT at the baseline of periodontal evaluation.

\section{Materials and Methods}

In addition, the radiographic signs of SOT (Figure 1) include:

1) An increased width of periodontal space, often with thickness of lamina dura along the lateral aspect of the root, in the apical lesion, and in buccal furcation areas;

2) a "vertical" or angular bony defects, rather than a "horizontal" destruction of the interdental septum;

3) radiolucence and condensation of the alveolar bone; and 4) root resorption. 5). tooth mobility [9].

The criteria of present specimens selected on the tooth of individuals affected SAP with SOT is according to the both of clinical signs and radiographic signs [9].

A total of 1013 teeth (83 maxillary molars and 367 non-molars) and 563 (114 mandibular molars and 449 non-molars) were randomly selected from a total of 53 individuals, (29 males and 24 females), who reporting or referred to the Periodontal Clinics of Dental School, Kaohsiung Medical University from 1981 to 2001. Those individuals ranging in age from 24 to 66 years (mean age $37.7 \pm 11.6$ years), affected severe advanced periodontitis (SAP) with SOT as the experimental group, whereas the SAP without SOT as the control group. Clinical examination of periodontal charting comprised the

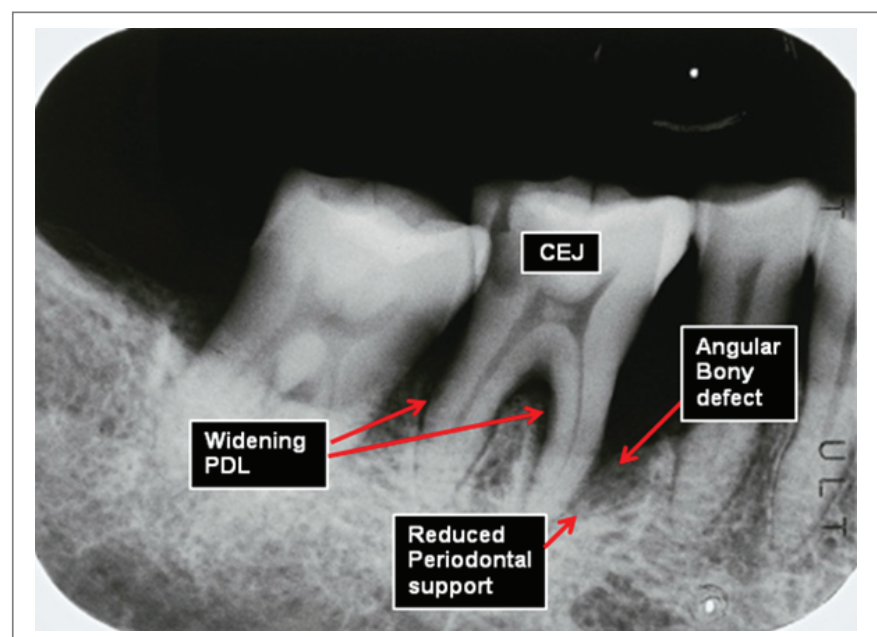

Figure 1: Shows that Radiographic images features of SOT included: angular bone defect, reduced periodontal support, increased tooth mobility, widening of PDL. age, sex, and a questionnaire of dental history including causes of missing tooth, gingival index (GI) [10], plaque index (PI) [11], initial probing pocket depths (PPD) and clinical attachment level (CAL). Routine 14-18 periapical radiographs including tooth distortion, poor radiographic qualitative, restoration obliterating the cement-enamel junction (CEJ), and overlapping in either teeth or CEJ measurements, which produced unreadable measurement points, were excluded from the study samples.

\section{Measurement of ARABL}

The proximal ARABL of mesial and distal tooth surfaces were examined at 10X magnification for alveolar bone loss using the digital scanning radiographic image analysis (DSRIA) [12]. Proximal ARABL was defined as bone defects of at least $2 \mathrm{~mm}$ distance between the CEJ and the alveolar crest. The radiographic CEJ, alveolar crest and root apex were used as three reference points for calculating ARABL (Figure 2).

Data analysis: The means and standard deviations of each tooth from the cases involved SAP with and without SOT, were calculated. The two-sample t-test for paired data was used to analyze the differences between different disease types, tooth with and without SOT, and ARABL at the baseline of periodontal charting.

\section{Reliability of the DSRIA}

The reliability was established by double reading of 20 teeth from 10 individuals randomly selected from a total 53 cases. All measurements were performed by the principal examiner and by a post graduate student, with 3 weeks between readings. The means and standard deviations of ARABL measured by DSRIA for each tooth were compared using reliability coefficients. Statistical significance was defined at $\mathrm{p}<0.05$.

Data analysis: The means and standards deviations of each tooth of both arches from the cases affected SAP with and without SOT were calculated. The two sample t-test for pair data was used to analyze the

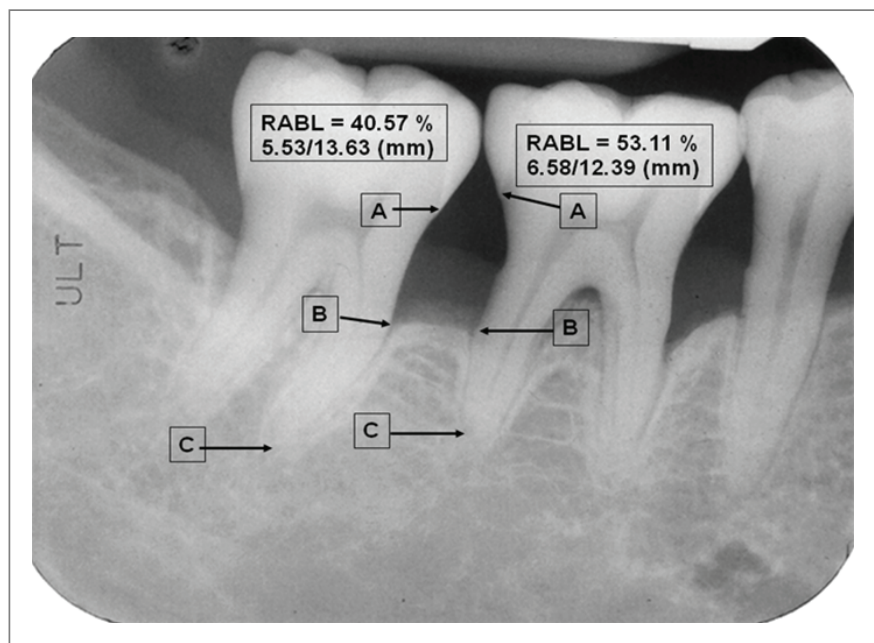

Figure 2: Proximal digital radiographic alveolar bone loss (DRABL) was defined as bone defects of at least $2 \mathrm{~mm}$ distance between the CEJ and the alveolar crest ( $2 \mathrm{~mm}$ under $\mathrm{CEJ}=\mathrm{A}$ ). The radiographic $\mathrm{CEJ}$, alveolar crest (B) and root apex (C) were used as three reference points for calculating $\mathrm{RABL}$.

\# 47: $A B=5.53 \mathrm{~mm} / \mathrm{AC}=13.63 \mathrm{~mm} ; \# 46: A B=6.58 \mathrm{~mm} / \mathrm{AC}=12.39 \mathrm{~mm}$

$A B / A C=40.57 \% \quad A B / A C=53.11 \%$ 
differences between teeth with and without SOT in both maxillary and mandibular arches $(\mathrm{p}<0.001)$ at the baseline (Table 1$)$, respectively.

\section{Results}

The interexaminer reliability coefficients using DSRIA [12] were $0.992,0.995$, and 0.986 for the maxillary arch, the mandibular arch, and both arches, respectively (Table 1). A similar finding was noted for intraexaminer reliability coefficients, where the reliability coefficients, with repeated measurements undertaken at a 3-week interval, in the maxillary, mandibular and both teeth were $0.992,0.994$ and 0.986 , respectively (Table 1).

Similar results were noted for intraclass and interclass reliability coefficients of maxillary, mandibular and both teeth and indicated that p-value $<0.001$ (Table 1).

Table 2 demonstrated that the differences between the ARABL ( $\mathrm{mm} / \mathrm{yr}$.) of teeth with and without SOT in both the maxillary and mandibular teeth. Results indicated that the ARABL on both the maxillary and mandibular teeth with SOT $(-0.26 \pm 0.14 \mathrm{~mm} / \mathrm{yr}$. $)$ and $(-0.20 \pm 0.10 \mathrm{~mm} / \mathrm{yr}$.) were greater loss than teeth without SOT $(-0.14$ $\pm 0.09 \mathrm{~mm} / \mathrm{yr})$ and $(-0.12 \pm 0.06 \mathrm{~mm} / \mathrm{yr}$.), respectively. The means (SD) of ARABL (mm/yr.) was $-0.26 \pm 0.14 \mathrm{~mm} / \mathrm{yr}$. (66 teeth) and -0.14 $\pm 0.09 \mathrm{~mm} / \mathrm{yr}$. (384 teeth), respectively, on the teeth with and without SOT in the maxillary teeth. The differences of means of ARABL on the maxillary teeth with SOT was significantly greater $(\mathrm{p}<0.0001)$ than teeth without SOTs (Table 2). Similarly, the comparison of differences the means (SD) of ARABL (mm/yr.) on the mandibular teeth with SOT $(-0.20 \pm 0.10 \mathrm{~mm} / \mathrm{yr}$. $)$ was significantly greater $(\mathrm{p}<0.0001)$ those teeth without SOT $(-0.12 \pm 0.06 \mathrm{~mm} / \mathrm{yr})$, respectively.

Table 3 showed that the means of ARABL on the maxillary (27 molars) with SOT $(-0.29 \pm 0.15 \mathrm{~mm} / \mathrm{yr}$.) and molars without SOT (56

Table 1: The reliability coefficient of inter-examiner and intra-examiner measurements of ARABL of teeth for maxillary, mandibular and both arches of teeth with SAP using DSRIA.

\begin{tabular}{|c|c|c|}
\hline Tooth location & Reliability coefficients & p-value \\
\hline \multicolumn{3}{|c|}{ Interexaminer } \\
\hline Maxillary tooth & 0.992 & $<0.001$ \\
\hline Mandibular tooth & 0.995 & $<0.001$ \\
\hline Both arch teeth & 0.986 & $<0.001$ \\
\hline \multicolumn{3}{|c|}{ Intraexaminer } \\
\hline Maxillary tooth & 0.992 & $<0.001$ \\
\hline Mandibular tooth & 0.994 & $<0.001$ \\
\hline Both arch teeth & 0.986 & $<0.001$ \\
\hline
\end{tabular}

Table 2: The differences of ARABL ( $\mathrm{mm} / \mathrm{yr}$ ) in maxillary and mandibular teeth with SOT and without SOT in Individuals, respectively.

\begin{tabular}{|c|c|c|c|c|}
\hline Teeth & & ARABL $(\mathbf{m m} / \mathbf{y r}$ ) & & \\
\hline SOT & $\mathrm{n}$ & $\begin{array}{c}\text { Maxillary Teeth } \\
\text { Means( } \pm \text { SD) }\end{array}$ & $\mathrm{n}$ & $\begin{array}{c}\text { Mandibular Teeth } \\
\text { Means( } \pm \text { SD) }\end{array}$ \\
\hline Teeth with SOT & 66 & $-0.26( \pm 0.14)$ & 66 & $-0.20( \pm 0.10)$ \\
\hline $\begin{array}{c}\text { Teeth without } \\
\text { SOT }\end{array}$ & 384 & $-0.14( \pm 0.09)$ & 497 & $-0.12( \pm 0.06)$ \\
\hline p-value & & $\mathrm{P}<0.0001$ & & $\mathrm{P}<0.0001$ \\
\hline
\end{tabular}

molars) $(-0.18 \pm 0.10 \mathrm{~mm} / \mathrm{yr}$.), respectively. Comparison of the means of ARABL on the maxillary molars with SOT was significantly greater loss $(\mathrm{p}<0.001)$ than those molars without SOT with two-sample t-test (Table 3).

The means (SD) of means of ARABL ( $\mathrm{mm} / \mathrm{yr}$.) on the mandibular molar with SOT (20 molars) and those molars without SOT (94 molars) were $-0.18 \pm 0.10 \mathrm{~mm} / \mathrm{yr}$. and $-0.12 \pm 0.07 \mathrm{~mm} / \mathrm{yr}$., respectively. The comparison of means of ARABL on the molars with SOT was significantly greater loss $(\mathrm{p}<0.05)$ than those molars without SOT $(\mathrm{p}<0.05)$ with two-sample t-test (Table 3$)$.

Results indicated that the means of ARABL on the maxillary nonmolar (48 non-molars) with SOT and those non-molars (328 nonmolars) without SOT were $-0.23 \pm 0.12 \mathrm{~mm} / \mathrm{yr}$. and $-0.13 \pm 0.08 \mathrm{~mm} /$ $\mathrm{yr}$, respectively. A comparison of means of ARPBL on non-molars with SOT was significantly greater loss $(\mathrm{p}<0.0001)$ than maxillary nonmolars without SOT using two-sample t-test (Table 4).

The means (SD) of ARABL ( $\mathrm{mm} / \mathrm{yr}$.) on mandibular non-molar with SOT were $-0.22 \pm 0.09 \mathrm{~mm} / \mathrm{yr}$. (46 molars), whereas non-molars without SOT (403 molars) were $-0.12 \pm 0.06 \mathrm{~mm} / \mathrm{yr}$., respectively. The difference of means of ARABL on the non-molars with SOT was significantly greater $(\mathrm{p}<0.0001)$ than those molars without SOT. With two-sample t-test (Table 4 ).

\section{Discussion and Conclusion}

This pilot study demonstrated that DSRIA resulted in a high value of correlation coefficients for both of intraexaminer $(r=0.994$, $\mathrm{p}<0.001)$ and interexaminer $(\mathrm{r}=0.995, \mathrm{p}<0.001)$ reliability tests to measure alveolar bone loss. Therefore, this pilot seems to be valuable as compare to those reports using traditional methods $[9,13]$.

Primary trauma from occlusion possible be caused by following reasons; a) alterations in occlusal forces and/or 2) reduced capacity of the periodontium to withstand occlusal forces $[13,14]$. Majority of the experimental animal studies on the effect of trauma from occlusion neither change the level of connective tissue attachment no initiation of pocket formation. Polson AM, et al. [15] indicated that no change of above reasons probably because the supracrestal gingival fibers are not

Table 3: The differences of ARABL (mm/yr.) on molars and non-molars with and without SOT in maxillary and mandibular arches.

\begin{tabular}{|c|c|c|c|c|}
\hline Tooth Types & $\mathbf{n}$ & $\begin{array}{c}\text { Maxillary Teeth } \\
\text { ARABL(mm/yr.) } \\
\text { Means( } \pm \text { SD) }\end{array}$ & $\mathbf{n}$ & $\begin{array}{c}\text { Mandibular Teeth } \\
\text { ARABL(mm/yr.) } \\
\text { Means( } \pm \text { SD) }\end{array}$ \\
\hline Molars with SOT & 27 & $-0.29( \pm 0.15)$ & 20 & $-0.18( \pm 0.10)$ \\
\hline Molars without SOT & 56 & $-0.18( \pm 0.14)$ & 94 & $-0.12( \pm 0.07)$ \\
\hline p-value & & $\mathrm{P}<0.001$ & & $\mathrm{P}<0.05$ \\
\hline
\end{tabular}

Table 4: The differences of ARABL (mm/yr.) on non-molars with and without SOT in both the maxillary and mandibular arches.

\begin{tabular}{|l|c|c|c|c|}
\hline \multicolumn{1}{|c|}{ Tooth Types } & $\mathbf{n}$ & $\begin{array}{c}\text { Maxillary Teeth } \\
\text { ARABL (mm/yr.) } \\
\text { Means( } \pm \text { SD) }\end{array}$ & $\mathbf{n}$ & $\begin{array}{c}\text { Mandibular Teeth } \\
\text { ARABL (mm/yr.) } \\
\text { Means( } \pm \text { SD) }\end{array}$ \\
\hline Non-molars with SOT & 48 & $-0.23( \pm 0.12)$ & 46 & $-0.22( \pm 0.09)$ \\
\hline Non-molars without SOT & & $-0.13( \pm 0.08)$ & 403 & $-0.12( \pm 0.06)$ \\
\hline p-value & & $P<0.0001$ & & $P<0.0001$ \\
\hline
\end{tabular}


affected and therefore prevent the apical migration of the junctional epithelium.

Secondary cause of periodontal destruction of alveolar bone loss is the most common cause of SOT because of the burden force increasing on the less remaining tissues and heavy leverage on the weaken periodontium $[16,17]$.

The most common clinical sign of SOT to the periodontium is increased tooth mobility. In the injury stage of SOT there is destruction of periodontal fibers, which will increase the mobility of the tooth. In the final stage the accommodation of the periodontium to increased forces entails a widening of the periodontal ligament, which also leads to increased tooth mobility. When it becomes progressively worse, it can be considered pathologic. Other causes of increased tooth mobility include advanced bone loss, inflammation of the PDL of periodontal or periapical origin [9].

In addition, the radiographic signs of SOT include 1) An increased width of periodontal space, often with thickness of lamina dura along the lateral aspect of the root, in the apical lesion, and in buccal furcation areas; 2) a "vertical" or angular bony defects, rather than a "horizontal" destruction of the interdental septum; 3) radiolucence and condensation of the alveolar bone; and 4) root resorption; 5) tooth mobility [9].

The criteria of present specimens selected on the tooth of individuals affected SAP with SOT is according to the both of clinical signs and radiographic signs [9].

Little or limited information regarding the differences between the individuals affected SAP with and without SOT was presented. Our results indicated that the ARABL on both the maxillary and mandibular teeth with SOT was greater loss than teeth without SOT with a significant difference, respectively. Results also showed that the ARABL on both the maxillary and mandibular non-molars with SOT was greater loss than teeth without SOT with a significant difference, respectively (Table 3 ).

This is the first report to address the alveolar bone lose rate per year of naturally progressing SAP affected SOT using the DSRIA in Taiwanese subjects at the baseline data. Little or limited studies have reported measurement differences of annually radiographic alveolar bone loss (ARABL) between the teeth affected SAP with SOT and the teeth without SOT at the baseline.

The differences of means of ARABL on the teeth with SOT was significantly greater $(\mathrm{p}<0.0001)$ than teeth without SOTs (Table 2$)$. Similarly, the comparison of differences the means (SD) of ARABL $(\mathrm{mm} / \mathrm{yr}$.) on the mandibular teeth with SOT $(-0.20 \pm 0.10 \mathrm{~mm} / \mathrm{yr}$. $)$ was significantly greater $(\mathrm{p}<0.0001)$ those teeth without SOT $(-0.12 \pm 0.06$ $\mathrm{mm} / \mathrm{yr}$ ), respectively. Therefore, result concluded that the means of ARABL of both maxillary and mandibular teeth with SOT remarkable and significantly greater $(\mathrm{p}<0.0001)$ than those teeth without SOT.

In addition, the conclusion also showed that the comparison of means of ARABL on the mandibular molars with SOT was significantly greater loss $(\mathrm{p}<0.05)$ than those molars without SOT $(\mathrm{p}<0.05)$ (Table 3). Similar trend of means of ARABL was also found on both of molars and non-molars, irrespective, of maxillary and mandibular arches areas (Table 4).

However, there has been little or no literatures regarding the yearly periodontal bone loss of severe advanced periodontitis associated with secondary trauma from occlusion and related to analysis using the DSRIA. Therefore, limited information about SAP with SOT to compare with early very few or no literatures.

Lindhe J \& Nyman S [18] and Rosling B, et al. [19] reported that the employment of proper periodontal therapy did not diminish the increased tooth mobility. Teeth affected SOT might still exhibit a progressively increased mobility and splinting of these teeth might necessary using therapeutic provisional prosthesis either uniterally or bilaterally cross arch design.

A recent case series study [20] used simple intentional replantation for periodontally compromised or hopeless teeth accompanied by extensive bone loss even beyond the root apex, and promising bone gain (without bone graft) was observed. An $88.2 \%$ overall cumulative survival rate of those affected teeth (5.1-13 years follow-up) was noted.

The other more recent study [21] involves a more long term followup case series regarding the preservation of similarly compromised teeth affected SAP with SOT using the strategies of non-surgical periodontal therapy (NSPT), therapeutic provisional prosthesis, and crown and sleeve-coping telescopic denture (CSCTD) were made to resolve SOT and stabilized the teeth affected SAP with SOT. These therapies were tried to hopefully achieve, bone fills of intrabony osseous defects of the candidate tooth sites. Supportive periodontal maintenance therapy was performed once every 2-3 months, throughout the end study. Additionally, clinical parameters and radiographic follow-up examinations of full mouth once every 1-2 years to monitor the periodontal bone regeneration of horizontal and angular bony defects.

\section{References}

1. Lang $\mathrm{N}$, Bartold PM, Cullinan M, Jeffcoat M, Mombelli A, et al. (1999) Consensus report: aggressive periodontitis. Ann Periodontol 4: 53-59.

2. Suomi JD, Plumbo J, Barbano JP (1968) A comparative study of radiographys and pockets measurements in periodontal disease evaluation. J Periodontol 39: 311-315.

3. Kelly GP, Cain RJ, Knowles JW, Nissle RR, Burgett FG, et al. (1975) Radiographs in clinical periodontal trials. J Periodontol 46: 381-386.

4. Rohner F, Cimasoni G, Vuagnat P (1983) Longitudinal radiographic study on the rate of alveolar bone loss in patients of a dental school. J Clin Periodontol 10: 643-651.

5. Becker W, Berg ML, Becker BE (1979) Untreated periodontal disease: A longitudinal study. J Periodontol 50: 234-244.

6. Bossert W, Marks HH (1956) Prevalence and characteristics of periodontal disease in 128000 persons under periodic dental observation. J Am Dent Assoc 52: 429-442.

7. Saari JT, Hurt WC, Biggs NL (1968) Periodontal bony defects in the dry skull. J Periodontol 39: 278-283.

8. Burmeister JA, Best AM, Palcanis KG, Caine FA, Ranney RR (1984) Localized juvenile periodontitis and generalized severe periodontitis: clinical findings. J Clin Periodontol 11: 181-192.

9. Boyle Jr WD, Via WF, McFall Jr WT (1973) Radiographic analysis of alveolar crest height and age. J Periodontol 44: 236 - 243.

10. Loe H, Silness J (1963) Periodontal disease in pregnancy. I. Prevalence and severity. Acta Odontol Scand 21: 533-551.

11. Silness J, Loe H (1964) Periodontal disease in pregnancy. II. Correlation between oral hygiene and periodontal condition. Acta Odontol Scand 22: 122-135. 
12. Hou GL, Hung CC, Yang YS, Shieh TY, Tsai CC (2003) Radiographic alveolar bone loss in untreated Taiwan Chinese subjects with adult periodontitis measured by the digital scanning radiographic image analysis method. Dentomaxillofac Radiol 32: 104-108.

13. Selikowitz HS, Sheiham A, Albert D, Williams GM (1981) Retrospective longitudinal study of the rate of alveolar bone loss in humans using bite-wing radiographs. J Clin Periodontol 8: 431-438.

14. Glickman I, Carranza FA (1990) Glickman's Clinical Periodontology. $7^{\text {th }}$ Edition, Saunders Publishers 264-285.

15. Polson AM, Meitner SW, Zander HA (1976) Trauma and progression of marginal periodontitis in squirrel monkeys. III Adaption of interproximal alveolar bone to repetitive injury. J Periodont Res 11: 279-289.

16. Glickman I, Smulow JB, Moreau J (1966) Effect of alloxan diabetes upon the periodontal response to excessive occlusal forces. J periodontal 37: 146-155.

17. Stahl SS, Miller SC, Goldsmith ED (1957) The effects of vertical occlusal trauma on the periodontium of protein deprived young adult rats. J Periodontol 28: 87-97.
18. Lindhe J, Nyman S (1975) The effect of plaque control and surgical pocket elimination on the establishment and maintenance of periodontal health. A longitudinal study of periodontal therapy I cases of advanced disease. J Clin Periodontol 2: 67-79.

19. Rosling B, Nyman A, Lindhe J (1976) The effect of systematic plaque control on bone regeneration in infra-bony pockets. J Clin Periodontol 3: 38-53.

20. Hou GL, Hou LT, Weisgold A (2016) Survival rate of Teeth with periodontally hopeless prognosis after therapies with intentional replantation and perioprosthetic procedures-a study of case series for 5-12 years. Clin Exp Dent Res 2: 85-95

21. Hou GL, Hou LT (2019) Therapeutic outcomes using the Sandwich's technique in treating severe advanced periodontitis with secondary occlusal trauma: A long-term study for 5.1-39 years. Int J Dent and Oral Heal 5: 64-73. 